\title{
Immunohistochemical Expression of MMP-2 in Pancreatic Carcinoma and Chronic Pancreatitis
}

\author{
Afaf Taha El-Nashar ${ }^{1}$, Eman Muhammad Salah Muhammad ${ }^{1}$, Mohammed Hamdy Sayed ${ }^{2}$, \\ Said Abuel-kheer Mohammed ${ }^{2}$ \\ ${ }^{1}$ Department of Pathology, Sohag University, Sohag, Egypt \\ ${ }^{2}$ Department of Pathology, El-Azhar University, Assiut, Egypt \\ Email address: \\ Elnasharafaf@yahoo.com (A. T. El-Nashar)

\section{To cite this article:} \\ Afaf Taha El-Nashar, Eman Muhammad Salah Muhammad, Mohammed Hamdy Sayed, Said Abuel-kheer Mohammed. \\ Immunohistochemical Expression of MMP-2 in Pancreatic Carcinoma and Chronic Pancreatitis. International Journal of Immunology. \\ Vol. 9, No. 3, 2021, pp. 47-53. doi: 10.11648/j.iji.20210903.12
}

Received: March 6, 2021; Accepted: March 16, 2021; Published: August 19, 2021

\begin{abstract}
Introduction: Matrix metalloproteinase (MMP)-2 is a member of MMPs family which is zinc-dependent endopeptidases that degrade all components of ECM and vascular basement membrane. MMPs are closely involved in tumor invasion and metastasis. Aim of the work: is to evaluate the immune-expression and clinical significance of MMPs molecule in patients with pancreatic carcinoma. Materials and Methods: A total of 25 pancreatic carcinomas were evaluated for expression of MMP-2 by immunohistochemical technique. The expression was evaluated. The study included 24/25 cases of PDAC and $1 / 25$ acinar cell carcinoma. PDAC included 5/24 cases of well differentiated, 16/24 cases of moderately differentiated, 3/24 cases of poorly differentiated adenocarcinoma. Results: Pancreatic carcinoma specimens showed positive cytoplasmic MMP-2 staining with high expression in $88 \%$ of studied specimens as their IRS ranged from 6/9 to 9/9 with high extent and intensity. There was a statistically significant relationship between MMP-2 expression and high grade in PDAC $(\mathrm{p}<0.024)$. Conclusion: MMP-2 was expressed in PDAC and not in pancreatitis cases. The expression of MMP-2 was correlated with high histological grade and poor prognosis in PDAC.
\end{abstract}

Keywords: (MMP-2) Matrix Metalloproteinases, (PDAC) Pancreatic Duct Adenocarcinoma, Immunohistochemistry, ECM Extracellular Matrix

\section{Introduction}

Pancreatic ductal adenocarcinoma (PDAC) is a highly fatal disease, usually diagnosed in an advanced stage which gives a slight chance of recovery. Pancreatic cancer is characterized by a rapid course, poor prognosis, and high mortality since most patients are diagnosed with metastases to lymph nodes and distant organs [1].

The early symptoms of pancreatic cancer are often very vague. They may precede the diagnosis by years and go unrecognized. This makes pancreatic cancer one of the cancers with the worst survival rates [2].

Pancreatic cancer is an intractable malignancy and is the seventh leading cause of global cancer deaths in industrialized countries. Based on GLOBOCAN 2018 estimates, pancreatic cancer is the 11th most common cancer in all over the world with estimated 458,918 new cases and 432,242 deaths $(4.5 \%$ of all cancer-related deaths) in 2018. The incidence and mortality of pancreatic cancer correlate with increasing age and is slightly more common in men than in women [3].

As the most common and most important pancreatic cancer, with rapid mortality and now also as the third leading cause of cancer-related deaths in the United States, PDAC has become synonymous with "pancreas cancer" [4].

In Egypt, pancreatic cancer has traditionally been considered rare, but the population in Egypt exhibits an unusually high rate of young-onset pancreatic cancer of 2.6 in females and 4.8 in males/ ASR/100000 population. The incidence rate for both sexes increases with age. Pancreatic cancer is seldom diagnosed before 55 years of age, and it can be defined as a disease of elderly populations because the highest incidence is reported in people over 70 years [5].

Currently, early diagnosis and radical surgery provide the 
only chance of long-term survival for patients with pancreatic carcinoma. Although there have been an improvement in the management of patients with pancreatic carcinoma, In the recent years, the 5-year overall survival rate of $\mathrm{PC}$ has remained low, at 3\% after effective treatment [6].

Local invasion and distant metastasis are the important biological characters of pancreatic carcinoma, occurring in early stage of the disease, causing the reduction of chance for surgery and death of the patients. Tumor infiltration and metastasis is a complex mechanism involved in a variety of cellular multi-step processes, among which the proteolytic degradation of extracellular matrix (ECM) is an essential event [7].

PDAC is characterized by a prominent desmoplastic reaction, results in formation of tumor microenvironment, consisting of a dense stroma surrounding the neoplastic cells [8].

The stroma forms the bulk of the tumor, taking up to $80-90 \%$ of the total tumor mass and consists of many components as myofibroblasts, macrophages, blood vessels and extracellular matrix components such as collagen I, collagen IV, laminin and fibronectin [9].

In the stroma, the extracellular matrix has been considered a stable structure that plays a supportive role in maintaining tissue morphology. However, it is evident that the extracellular matrix forms the fundamental role on the surrounding cells. According to this concept, loss of extracellular matrix homeostasis and integrity is considered one of the hallmarks of cancer and typically defines transitional events, resulting in cancer progression and metastasis [10].

MMPs have long been held responsible for cancer cell invasion and metastasis, because of the great impact of metalloproteinases in remodelling the ECM of tumours. MMPs and their proteolytic function are responsible for several steps of cancer as cancer-cell growth, differentiation, apoptosis, migration, invasion, and tumours angiogenesis. They have significant roles in initial stages of cancer progression by inducing genomic instability and DNA damage as well as invasion and metastasis by facilitating invasion of cancer cells by ECM degradation [11, 12].

MMP-2 is an important member of the MMPs family which plays a significant part in the development of some neoplastic diseases [13].

Matrix metalloproteinase (MMP)-2 is a member of MMPs family which is zinc-dependent endopeptidases that degrade all components of ECM and vascular basement membrane. They play an important part in the development of numerous neoplasms and connective tissue diseases. MMPs are closely involved in tumour invasion and metastasis [14].

The aim of this study: is to evaluate immunohistochemical expression of MMP-2 in pancreatic carcinoma, its correlation with the established prognostic factors.

\section{Materials and Methods}

Twenty five formalin-fixed paraffin-embedded tissue blocks of pancreatic carcinoma and five tissue blocks of chronic pancreatitis as a control group were retrieved for this study from the archived material of the Laboratory of Pathology Department, Sohag University Hospitals and Sohag Oncology Center during the period from 2015 to 2018. The study protocol was approved from the Institutional Ethical Committee of Sohag University. Tumor tissue samples were obtained by excisional biopsy of pancreatic masses and Whipple operation (pancreatico-duodenectomy). Clinico-pathological data of the patients in the study as age, sex, tumor size, and the presence of distant metastasis were retrieved from patients' medical files.

Four-micron-thick tissue sections from the tumours were prepared from formalin-fixed, paraffin-embedded tissue blocks and stained with Hematoxylin and Eosin for histological type, grade, perineural invasion, lympho-vascular invasion and presence of regional lymph node metastasis. Tumor grade and pathological stage were evaluated according to WHO recommendations [15].

\subsection{Immunohistochemistry}

Immunostaining method using the peroxidase-labeled streptavidin-biotin technique to detect MMP-2 was done. Three-micron-thick tissue sections mounted on Poly-Lysine coated slides were de-paraffinized and rehydrated. Endogenous peroxidase activity was blocked using peroxidase blocking reagent (Cat \# TP-015-H, Lab Vision Corporation). The antigen sites were unmasked by immersing the slides in sufficient amounts of $10 \mathrm{Mmol}$ sodium citrate buffer, $\mathrm{pH}$ 6.0. Tissue sections were incubated in a microwave for 10-15 min, allowed to cool down for $20 \mathrm{~min}$, washed in distilled water, then in phosphate-buffered saline (PBS, pH 6.0). Tissue sections were incubated in normal goat serum to block nonspecific interactions. Tissue sections were incubated overnight at room temperature with MMP-2 rabbit polyclonal antibody (25 $\mu \mathrm{m}$, concentrate, Cat\#42599, GeneTex Corporation) at a dilution of $1 / 100$. The resulting immune-complex was detected by a universal staining kit (Cat \# TP-015-H, Lab Vision Corporation). Tissue sections were treated with biotinylated polyvalent goat anti-mouse secondary antibody (Lab Vision Corporation), and then peroxidase-labeled streptavidin was applied for 10-15 min at room temperature, rinsed in PBS, incubated with 14-diaminobenzidine for $5 \mathrm{~min}$. The tissue sections were counter-stained in Mayer's Hematoxylin, washed in tap water, dehydrated in alcohol, cleared in Xylene, left to dry, then mounted with DPX, and cover slipped. Breast carcinoma was used as positive control for MMP-2 antibody.

\subsection{Evaluation of MMP-2 Protein Expression}

MMP-2 protein expression was semi-quantitatively evaluated according to the percentage and the intensity of stained cells. The percentages of stained cells were divided as follows: 0 for no staining; 1 point for $<10 \% ; 2$ points for $10-30 \% ; 3$ points for $>30 \%$ of stained cells. The intensity of staining was ranged as following: 0 , absent; 1, weak; 2, moderate; 3 , strong. The percentage and intensity of staining were multiplied to produce an immunoreactivity score (IRS) 
for each case. A final total score $\leq 3$ was defined as low expression, and $>3$ was determined to be high expression [14]. Data analysis were done using SPSS version 22. Data were presented as number, percentage, mean \pm standard deviation (SD), and median. The correlation between MMP-2 expression and clinico-pathological data were determined using a Mann-Whitney test for quantitative variables and a Kruskal Wallis test for qualitative variables. Spearman correlation was done to measure the correlation between quantitative variables. P-value was considered statistically significant when $\mathrm{p}<0.05$.

\section{Results}

\subsection{Clinical Data and Pathological Characteristics of the Studied Specimens}

The age range of the 25 patients with pancreatic carcinoma were 40 to 69 years old with mean \pm SD was $56.32( \pm 8.57)$ and the median age was 59 years old. Patients with pancreatic carcinoma included in this study were 14 males and 11 females. More than half of the patients with pancreatic carcinoma (56\%) were clustered in the age group less than 60 years old. The size of tumor masses ranged between $1-7 \mathrm{~cm}(6$ specimens $\leq 2 \mathrm{~cm}$ and 19 specimens $>2 \mathrm{~cm}$ ). Specimens was taken either by Whipple operation (pancreatico-duodenectomy) or by excisional biopsy of pancreatic masses. Most of these tumour masses (18/25) were located at the pancreatic head and the rest $(7 / 25)$ were located at the pancreatic body and tail.

On histopathological typing; 24/25 of the specimens were PDAC and 1/25 was acinar cell carcinoma. Regarding tumor differentiation, PDAC included $5 / 24$ cases of well differentiated adenocarcinoma, 16/24 cases of moderately differentiated adenocarcinoma, 3/24 cases of poorly differentiated adenocarcinoma. According to WHO staging system of pancreatic carcinoma [16], the studied cases has been classified pathologically into pT1, pT2, pT3 and pT4 in 6, 12, 6 and one case; respectively (Table 1).

Table 1. The clinicopathologicl features of the studies cases

\begin{tabular}{ll}
\hline Clinicopathological data & No \\
\hline Male & 14 \\
Female & 11 \\
Histological type & \\
PDAC & 24 \\
Well differentiated AC & 5 \\
Moderately differentiated AC & 16 \\
Poorly differentiated AC & 3 \\
Acinar cell carcinoma & 1 \\
WHO staging of the cases & \\
pT1 & 6 \\
pT2 & 12 \\
pT3 & 6 \\
pT4 & 1 \\
he tumour size & \\
$\leq 2$ cm & 6 \\
$>2$ cm & 19 \\
Tumour at head of pancreas & 18 \\
Tumour at the tail or body & 7 \\
\hline
\end{tabular}

\subsection{Immunohistochemical Features}

Pancreatic carcinoma specimens showed positive cytoplasmic MMP-2 staining with high expression in 22/25 studied specimens $(88 \%)$ as their IRS ranged from $6 / 9$ to $9 / 9$ with high extent and intensity. Only $3 / 25$ (12\%) of the studied cases showed positive MMP-2 staining with low MMP-2 expression. Well differentiated PDAC showed high MMP-2 expression at 4/5 specimens and low expression at $1 / 5$ specimen. Moderately differentiated PDAC showed high MMP-2 expression in 14/16 specimens and low expression in $2 / 16$ specimens. Poorly differentiated PDAC showed high MMP-2 expression at $3 / 3$ specimens. A specimen of acinar cell carcinoma showed high MMP-2 expression. The surrounding normal pancreatic tissue and five specimens of chronic pancreatitis showed negative MMP-2 staining. Stromal cells showed focal positive MMP-2 staining in 12/25 of specimens [Table 2, Figures 1 $\& 2]$. There was a statistically significant relationship $(\mathrm{p}<0.024)$ between MMP-2 expression and tumour grade in PDAC [Table 2 and Figure 1]. However, no statistical significant relationship was found between MMP-2 staining and other parameters; tumour size, lympho-vascular invasion, perineural invasion, lymph node metastasis, or tumour stage.

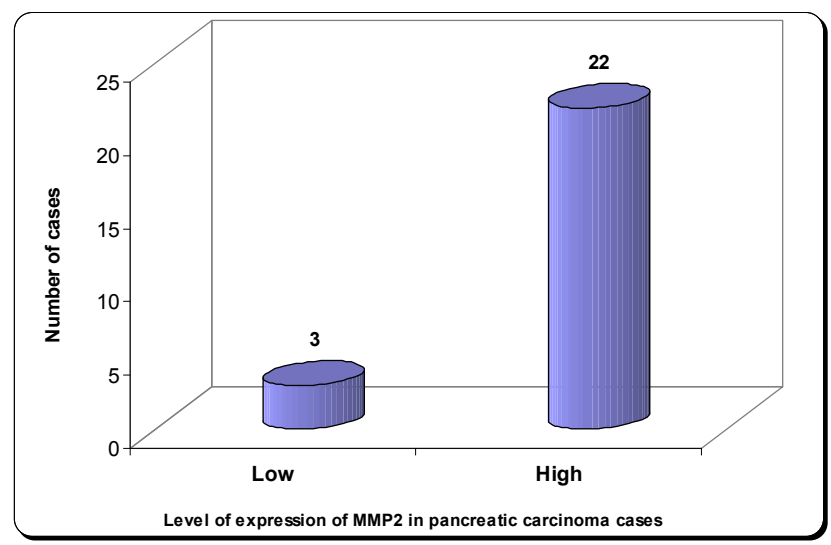

A

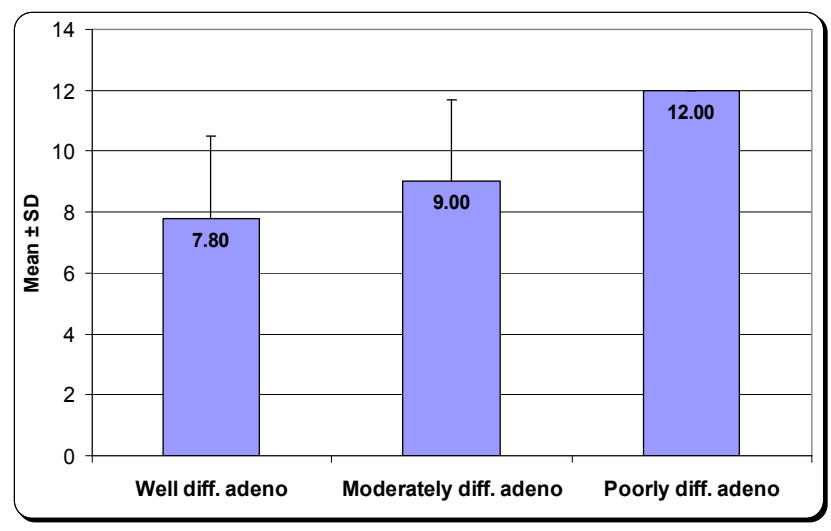

B

Figure 1. (A) $M M P-2$ expression in pancreatic carcinoma.(B) The relationship between MMP-2 expression and tumour grade. 


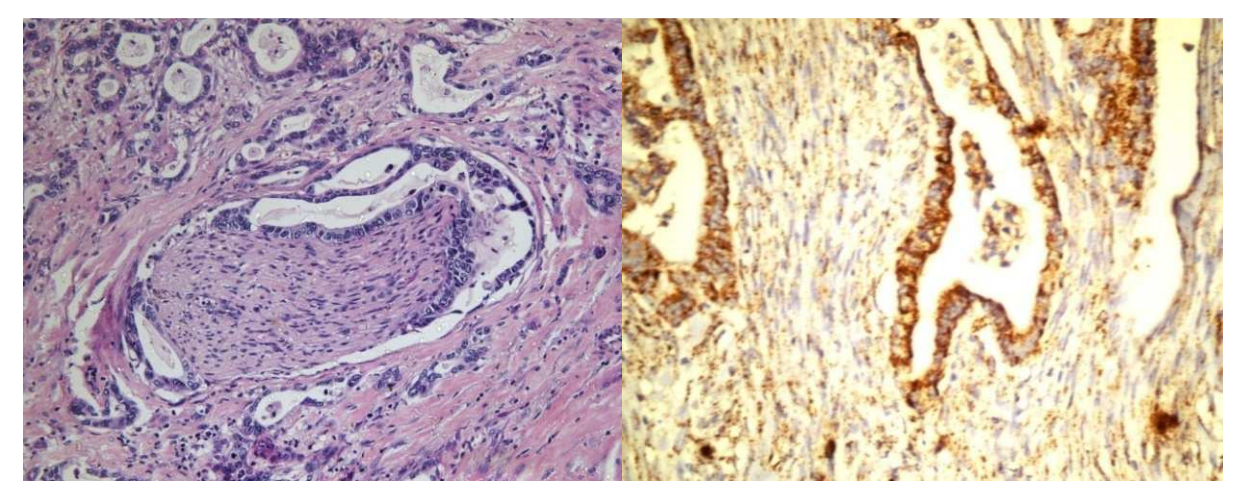

A

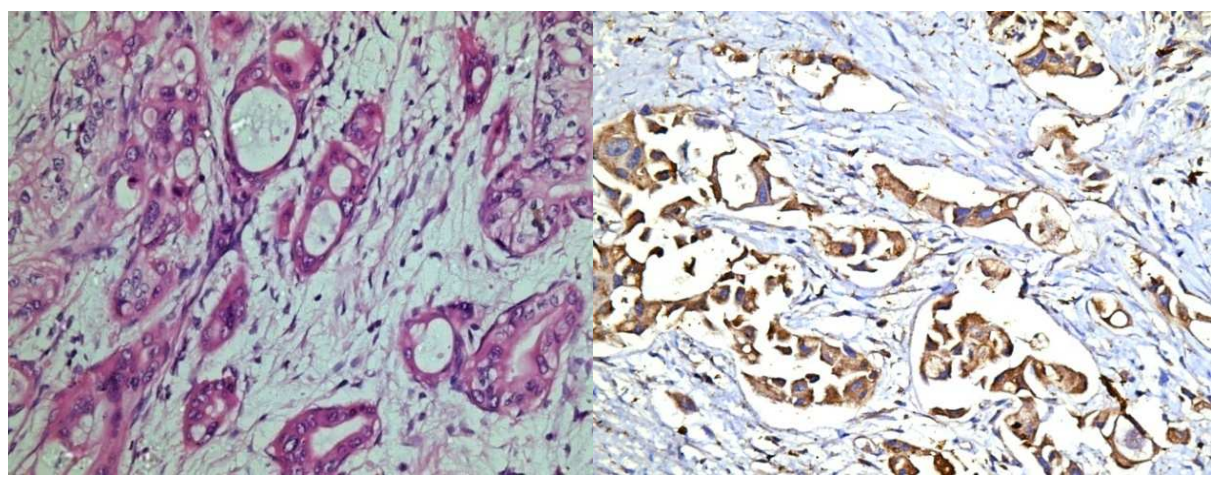

B

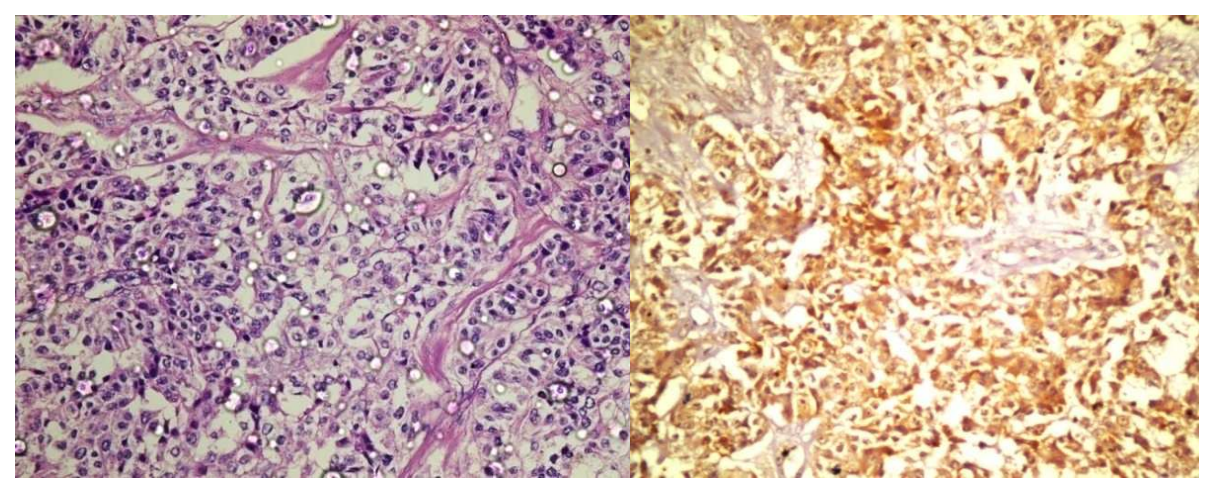

$\mathrm{C}$

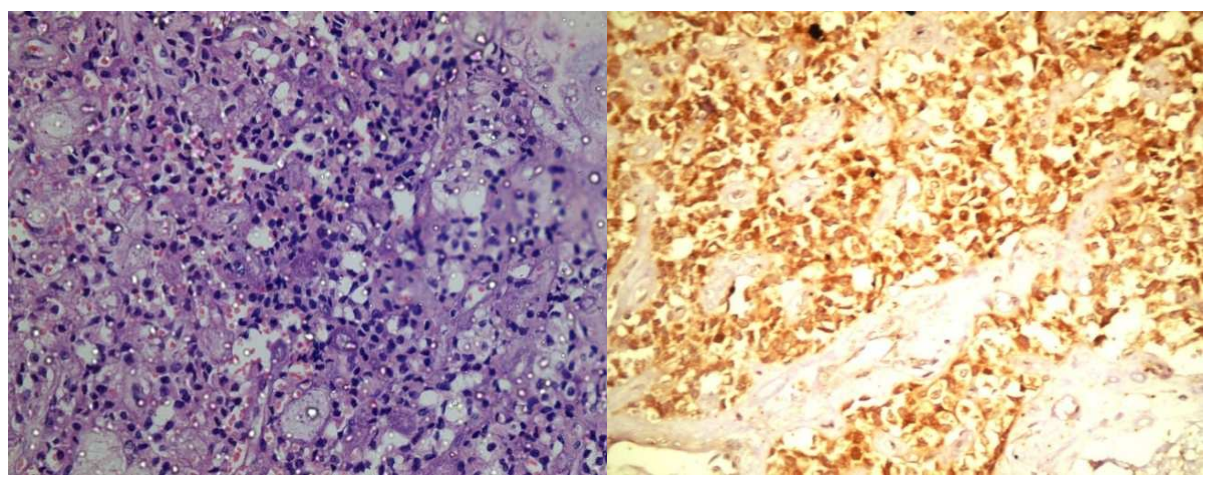

$\mathrm{D}$

Figure 2. Hematoxylin and eosin stained and MMP-2 cytoplasmic expression in PDAC.

(A) Well differentiated PDAC. (B) Moderately differentiated PDAC.

(C) Poorly differentiated PDAC. (D) Acinar cell carcinoma of pancreas. 
Table 2. Relationship between MMP-2 expression and clinico-pathological parameters.

\begin{tabular}{|c|c|c|c|c|c|c|}
\hline & \multirow{2}{*}{ No } & \multicolumn{2}{|c|}{ MMP-2 expression } & \multicolumn{2}{|c|}{ MMP2 Expression } & \multirow{2}{*}{ P-value } \\
\hline & & + & +++ & Mean \pm SD & Median (Range) & \\
\hline Histological grade: & 25 & 3 & 22 & & & \multirow{4}{*}{$0.024 *$} \\
\hline Well differentiated PDAC & 5 & 1 & 4 & $7.80 \pm 2.68$ & $9.0(3.0-9.0)$ & \\
\hline Moderately differentiated PDAC & 16 & 2 & 14 & $9.00 \pm 2.68$ & $9.0(3.0-12.0)$ & \\
\hline Poorly differentiated PDAC & 3 & - & 3 & $12.00 \pm 0.00$ & $12.0(12.0-12.0)$ & \\
\hline Lymph nodes: Positive & 13 & & & $9.23 \pm 2.28$ & $9.0(3.0-12.0)$ & \multirow{2}{*}{0.625} \\
\hline Negative & 12 & & & $9.25 \pm 3.25$ & $9.0(3.0-12.0)$ & \\
\hline Perineural invasion: Positive & 20 & & 17 & $8.85 \pm 2.83$ & $9.0(3.0-12.0)$ & \multirow{2}{*}{0.117} \\
\hline Negative & 4 & & & $10.80 \pm 1.64$ & $12.0(9.0-12.0)$ & \\
\hline Lympho-vascular invasion: Positive & 2 & & & $10.50 \pm 2.12$ & $10.5(9.0-12.0)$ & \multirow{2}{*}{0.499} \\
\hline Negative & 22 & & & $9.13 \pm 2.78$ & $9.0(3.0-12.0)$ & \\
\hline Size of tumour: $\leq 2 \mathrm{~cm}$ & & & & $10.00 \pm 1.55$ & $9.0(9.0-12.0)$ & \multirow{2}{*}{0.617} \\
\hline$>2 \mathrm{~cm}$ & & & & $9.00 \pm 3.00$ & $9.0(3.0-12.0)$ & \\
\hline Staging: T1/ T2 & 18 & & 15 & $9.17 \pm 3.17$ & $9.0(3.0-12.0)$ & \multirow{2}{*}{0.658} \\
\hline $\mathrm{T} 3 / \mathrm{T} 4$ & 7 & & 7 & $9.43 \pm 1.13$ & $9.0(9.0-12.0)$ & \\
\hline
\end{tabular}

\section{Discussion}

The current study was carried out on 25 formalin-fixed, paraffin-embedded tissue blocks of pancreatic carcinoma and 5 cases of chronic pancreatitis.

The mean age of the study patients was 56.32 years ranging from 40 to 69 years. Males were more frequently involved by pancreatic carcinoma than females. This was in agreement with the national reports of age incidence of pancreatic cancer at 2020 which stated that the risk of developing pancreatic cancer increases with age. Most people who develop pancreatic cancer are older than $45,90 \%$ are older than 55 and $70 \%$ are older than 65 . More men are diagnosed with pancreatic cancer than women according to American Cancer Society, 2020 [17].

Histopathological examination showed that 24 specimens were PDAC; $5 / 25$ cases of well differentiated, $16 / 25$ cases of moderately differentiated and $3 / 25$ cases of poorly differentiated pancreatic carcinoma and one specimen was acinar cell carcinoma. Perineural invasion was observed in $20 / 25$, while vascular invasion was observed only in $2 / 25$ of the studied tumours. Lymph node involvement was observed in 13/25 of the tumours. Regarding tumour stage, most cases of this study were classified as either pT2; $12 / 25$ cases or pT3; $6 / 25$ cases.

In the present study, immunohistochemical expression of MMP-2 was cytoplasmic in 22/25 (88\%) of cases of pancreatic carcinoma and the IRS was $9 / 9$ to $6 / 9$ with high extent and intensity. This expression was nearly similar to that reported by Zhai et al., who found that MMP-2 expression in pancreatic carcinoma was positive in 96/122 (78.7\%) [14], while Giannopoulos et al. reported that MMP-2 expression in pancreatic carcinoma cases were positive in 21/32 (65.6\%) of cases [18]. Jakubowska et al., reported that MMP-2 expression in pancreatic carcinoma cases were positive in 16/29 (55.17\%) [19]. The different values of MMP-2 expression in the aforementioned studies could be due to the larger number of cases and different antibodies used for MMP-2 detection in pancreatic carcinoma in their studies.

Non-neoplastic acinar parenchymal and pancreatic ductal epithelium was negative in all 25 cases in this study. This finding was similar to that observed by Roy et al. and Zhai et al., who reported that MMP-2 expression was higher in pancreatic carcinoma tissues than that in para-carcinoma tissues by IHC staining $[13,14]$. Studies by Jakubowska et al. reported that normal pancreatic acini and ducts were negative with MMP-2 [19].

Stromal cells showed positive staining in 12/25 (48\%) of pancreatic carcinoma cases in the current study. However, Jakubowska et al. documented that stromal cells showed positive staining in $(79.31 \%)$ of pancreatic carcinoma cases [19], while Giannopoulos et al. reported that most cases in his study showed stromal positive staining with MMP-2 [18].

In this study, there was a statistically significant relationship between MMP-2 expression and tumour grade $(\mathrm{p}<0.024)$. This finding was nearly similar to that of Giannopoulos et al. who found a significant correlation between MMP-2 expression in pancreatic carcinoma and the degree of tumour differentiation [18]. Also Zhai et al reported that MMP-2 expression in pancreatic carcinoma was significantly correlated with poor histological grade [14].

There was no statistically significant relation between MMP-2 expression and the clinico-pathological parameters of pancreatic carcinoma; tumour size, perineural invasion, lympho-vascular invasion, lymph node metastasis, or tumour stage and Slapak et al 2020 reported the same finding in a systematic review of the studies focusing on matrix metalloproteases in pancreatic cancer [20]. However, Jakubowska et al. 2016 found only significant correlation with female gender [19], whereas, Giannopoulos et al. reported a significant correlation with patients' age [18].

Of the studied pancreatic carcinoma with peri-neural invasion, 17/20 (85\%) cases showed high IHC-MMP-2 expression but with no statistical significant correlation. All cases with advanced stages ( 7 cases T3/T4) showed high MMP-2 expression and with the less advanced stages (15 cases T1/T2) showed high MMP-2 expression but with no significant correlation. This may indicate that MMP-2 is related to the conversion of the pancreatic acini from the normal to the malignant condition irrelevant to tumour stage. Zhai et al. reported that MMP-2 expression in pancreatic 
carcinoma was significantly correlated with perineural invasion, lymph node, distant metastasis, and advanced stage [14]. Absence of correlation between MMP-2 expression and most of the clinical or pathological characteristic of pancreatic carcinoma in the current study may be due to small sample size and incomplete follow up of the patients for a sufficiently longer period of time. There is increasing evidence indicating that MMP-2 has a critical role in tumour invasion and metastasis.

Some reports have shown that the prognostic significance of MMP-2 expression in several types of tumours. Vasala et al. and Yao et al demonstrated that overexpression of MMP-2 is significantly associated with a reduced survival rate and may be as an independent prognostic marker for bladder cancer [21, 22].

$\mathrm{Wu}$ et al. showed that MMP-2 expression has significant correlation with tumour differentiation, invasion and lymph node metastasis, and act as a significant prognostic marker in gastric carcinoma [23]. Dragutinović et al. reported that overexpression of MMP-2 is significantly correlated with tumor progression and might be useful to predict tumour recurrence in patients with colorectal adenocarcinoma [24]. Aparna et al. documented that a higher MMP-2 expression is associated with local recurrence, distant metastasis and shorter survival, and may serve as an indicator of poor prognosis in early stages of tongue SCC [25].

In the current study the chronic pancreatitis cases showed no MMP-2 expression and this contrasts with Choi et al and Manjari et al, who reported that the MMp-2 was expressed in pancreatitis but the intensity of MMP2 staining tended to be lower in the pancreatic ductal epithelial cells in autoimmune pancreatitis (AIP) and in alcoholic chronic pancreatitis ACP $[26,27]$.

\section{Conclusions}

MMPs and their proteolytic function are responsible for cancer-cell growth, differentiation, apoptosis, migration, invasion, and tumours angiogenesis. They have significant roles in invasion and metastasis by facilitating invasion of cancer cells by ECM degradation.

MMP-2 immune expression was positive in pancreatic carcinoma with various grades, but negative in chronic pancreatitis cases. There was a significant relationship between MMP-2 expression and poor histological grade in PDAC.

\section{References}

[1] Li C, Heidt DG, Dalerba P, Burant CF, Zhang L, Adsay V, Wicha M, Clarke MF and Simeone DM. "Identification of pancreatic cancer stem cells," Cancer Research, vol. 67, no. 3, pp. 1030-1037, 2007.

[2] Nai Q, Luo H, Zhang P, Hossain M. A, Gu P, Sidhom IW, Mathew T, Islam M, Yousif AM, Sen S. How Early Can Pancreatic Cancer Be Recognized? a case report and review of literature: Cancer statistics. Cancer J Clin 2014; 64: 9-29.
[3] Bray F, Ferlay J, Soerjomataram I, Siegel RL, Torre LA, Jemal A. Global cancer statistics 2018: GLOBOCAN estimates of incidence and mortality worldwide for 36 cancers in 185 countries. CA Cancer J Clin. 2018; 68 (6): 394-424.

[4] Mostafa ME, Erbarut-Seven I, Pehlivanoglu B, Adsay V. Pathologic classification of pancreatic cancers. Chin Clin Oncol 2017; 6: 59.

[5] SEER Cancer Statistics Review, 1975-2013. National Cancer Institute, Bethesda, MD. 2016.

[6] Siegel RL, Miller KD and Jemal A. Cancer statistics, 2019. CA Cancer J. Clin., https://doi.org/10.3322/caac.21551

[7] Binker MG, Binker-Cosen MJ, Binker-Cosen AA, Cosen-Binker LI. Microenvironmental factors and extracellular matrix degradation in pancreatic cancer. JOP 2014; 15: 280-285.

[8] Hidalgo M. Pancreatic cancer. N. Engl. J. Med. 2010, 362, 1605-1617.

[9] Bijlsma MF and Van Laarhoven HWM. The conflicting roles of tumor stroma in pancreatic cancer and their contribution to the failure of clinical trials: A systematic review and critical appraisal. Cancer Metastasis Rev. 2015, 34, 97-114.

[10] $\mathrm{Lu} \mathrm{P}$, Weaver VM, Werb Z. The extracellular matrix: A dynamic niche in cancer progression. J. Cell Biol. 2012, 196, 395-406.

[11] Yadav L, Puri N, Rastogi V, Satpute P, Ahmad R, Kaur G. Matrix metalloproteinases and cancer-Roles in threat and therapy. Asian Pac. J. Cancer Prev. 2014, 15, 1085-1091.

[12] Radisky E. S, Raeeszadeh-Sarmazdeh M, Radisky D. C. Therapeutic Potential of Matrix Metalloproteinase Inhibition in Breast Cancer. J. Cell Biochem. 2017, 118, 3531-3548.

[13] Roy, R, Zurakowski D, Wischhusen J, Frauenhoffer C, Hooshmand S, Kulke M and Moses MA. Urinary TIMP-1 and MMP-2 levels detect the presence of pancreatic malignancies. Br J Cancer. 2014; 111: 1772-1779.

[14] Zhai LL, Cail CY, Wu Y, Tang ZG. Correlation and prognostic significance of MMP-2 and TFPI-2 differential expression in pancreatic carcinoma, Int J Clin Exp Pathol 2015; 8: 682-691.

[15] Gonzalez RS. WHO classification. Pathology Outlines.com website.

https://www.pathologyoutlines.com/topic/pancreaswho.html. Accessed February 2nd, 2021.

[16] Dragovich T. Pancreatic cancer staging 2020, Oncology.

[17] American Cancer Society's (ACS) publication, Cancer Facts \& Figures 2020, and the ACS website (January 2020).

[18] Giannopoulos G, Pavlakis K, Parasi A, Kavatzas N, Tiniakos D, Karakosta A, Tzanakis N and Peros G. The Expression of Matrix Metalloproteinases-2 and -9 and their Tissue Inhibitor 2 in Pancreatic Ductal and Ampullary Carcinoma and their Relation to Angiogenesis and Clinico-pathological Parameters. Anticancer research, 2008; 28: 1875-1882.

[19] Jakubowska K, Pryczynicz A, Januszewska J, Sidorkiewicz I, Andrzej Kemona A, Niewiński A, Lewczuk L, Kędra B, and Guzińska-Ustymowicz K. Expressions of Matrix Metalloproteinases 2, 7, and 9 in Carcinogenesis of Pancreatic Ductal Adenocarcinoma. Hindawi Publishing Corporation, Disease Markers 2016; 1-7. 
[20] Slapak EJ, Duitman JW, Tekin C, Bijlsma MF and Spek CA. Matrix Metalloproteases in Pancreatic Ductal Adenocarcinoma: Key Drivers of Disease Progression? Biology 2020, (9), 80.

[21] Vasala K, Pääkkö P, Turpeenniemi-Hujanen T. Matrix metalloproteinase-2 immuno-reactive protein as a prognostic marker in bladder cancer. Urology 2003; 62: 952-957.

[22] Yao Z, Yuan T, Wang H, Yao S, Zhao Y, Liu Y, Jin S, Chu J, Xu Y, Zhou W, Yang S, Liu Y. MMP-2 together with MMP-9 overexpression correlated with lymph node metastasis and poor prognosis in early gastric carcinoma Tumour Biol. 2017 Jun; 39 (6): 1010428317700411.

[23] $\mathrm{Wu} \mathrm{ZY,} \mathrm{Li} \mathrm{JH,} \mathrm{Zhan} \mathrm{WH} \mathrm{and} \mathrm{He} \mathrm{YL.} \mathrm{Lymph} \mathrm{node}$ micrometastasis and its correlation with MMP-2 expression in gastric carcinoma. World J Gastroenterol 2006; 12: 2941-2944.

[24] Dragutinović VV, Radonjić NV, Petronijević ND, Tatić SB, Dimitrijević IB, Radovanović NS, Zoran V Krivokapić ZV.
Matrix metalloproteinase-2 and (MMP-9) in preoperative serum as independent prognostic markers in patients with colorectal cancer. Mol Cell Biochem 2011; 355: 173-178.

[25] Aparna M, Rao L, Kunhikatta V, Radhakrishnan R. The role of MMP-2 and MMP-9 as prognostic markers in the early stages of tongue squamous cell carcinoma. J Oral Pathol Med 2014.

[26] Choi EK, Kim MH, Jang SJ, Lee KH, Hwang CY, Moon SH, Lee TY, Koh CO, Park DH, Lee SS, Seo DW, Lee SK. Differences in pancreatic immunohistochemical staining profiles of TGF-beta1, MMP-2, and TIMP-2 between autoimmune and alcoholic chronic pancreatitis. Pancreas: October 2009 - Volume 38: (7): 739-745.

[27] Manjari KS, Nallari P, Venkateshwari A. Matrix metalloproteinases in pancreatitis: current insights. Metalloproteinases In Medicine, January 2017 Volume (4): 15-23. 